\title{
Purified vitexin compound 1 inhibits growth and angiogenesis through activation of FOXO3a by inactivation of Akt in hepatocellular carcinoma
}

\author{
JIANGANG WANG ${ }^{1}$, XINGXING ZHENG ${ }^{1}$, GUANGYAO ZENG ${ }^{2}$, YINGJUN ZHOU ${ }^{2}$ and HONG YUAN ${ }^{1,3}$ \\ ${ }^{1}$ Department of Cardiology, The Third Xiangya Hospital, Central South University; ${ }^{2}$ School of Pharmaceutical Science, \\ Central South University; ${ }^{3}$ Center of Clinical Pharmacology, The Third Xiangya Hospital, \\ Central South University, Changsha, Hunan 410013, P.R. China
}

Received August 21, 2013; Accepted December 2, 2013

DOI: $10.3892 / \mathrm{ijmm} .2013 .1587$

\begin{abstract}
Vitexins, isolated from the seeds of the Chinese herb Vitex negundo, is known to exert antitumor activity in cancer xenograft models and cell lines. The aim of the current study was to examine whether the Akt/forkhead box protein $\mathrm{O} 3 \mathrm{a}$ (FOXO3a) pathway mediates the biological effects of purified vitexin compound 1 (VB-1) in hepatocellular carcinoma (HCC) cells. The effect of VB-1 on the viability of the HCC cell lines HepG2, Hep3B, Huh-7 and the human embryonic liver cells L-02 was investigated using the 3-(4,5-dimethylthiazol-2-yl)-2,5-diphenyltetrazolium bromide (MTT) assay. Growth inhibition was assessed by clonogenic assay, and cell cycle arrest was investigated using flow cytometry. Inhibition of angiogenesis was evaluated using a matrigel in vitro HUVEC tube formation assay. The effects on the Akt/FOXO3a pathway were detected by western blotting. VB-1 suppressed the proliferation of HepG2, Hep3B, Huh-7 cells, but had little effect on L-02 cells. VB-1 inhibited anchorage-dependent and -independent HepG2 cell growth in a concentration-dependent manner by induction of cell cycle arrest at G1/G0. VB-1 also reduced the secretion of vascular endothelial growth factor (VEGF), resulting in the inhibition of endothelial tube formation. Phosphorylated Akt and its downstream effector FOXO3a were downregulated in VB-1treated HepG2 cells. Knockdown of Akt1 by small interfering RNA (siRNA) enhanced growth inhibition, and silencing FOXO3a by siRNA attenuated this action. VB-1 inhibited growth and induced cell cycle arrest at G1/G0 by regulating the Akt/FOXO3a pathway. The findings suggested that VB-1 is a potentially promising candidate for the treatment of HCC.
\end{abstract}

Correspondence to: Dr Hong Yuan, Department of Cardiology, The Third Xiangya Hospital, Central South University, Tongzipo 138, Changsha, Hunan 410013, P.R. China

E-mail: yuanhong01@vip.sina.com

Key words: hepatocellular carcinoma, purified vitexin compound 1, angiogenesis, Akt, forkhead box protein O3a, therapeutic action

\section{Introduction}

Hepatocellular carcinoma (HCC) is the most common cause of cancer mortality worldwide (1). Potentially curative treatments include hepatic resection, liver transplantation, microwave coagulation and radiofrequency ablation. Palliative therapy involves transarterial chemoembolization (TACE), systemic chemotherapy and targeted therapy with sorafenib $(2,3)$. Liver transplantation and surgical resection improve survival in patients with small, non-invasive and non-metastatic tumors. However, there are still no effective treatment for advanced disease $(4,5)$.

Lignans are a group of complex polyphenolic antioxidants found in plants. Clinical studies suggest that lignans are one of the most promising classes of dietary agents for cancer prevention $(6,7)$. A number of lignans have been investigated in preclinical tumor xenograft models, prospective and casecontrol epidemiologic studies, and in some clinical trials (6-10). In the absence of data from randomized clinical trials, it is not known whether lignans can reduce cancer growth. However, several biomarker-based neoadjuvant trials have demonstrated that dietary intake of flaxseed lignan inhibits tumor cell proliferation and induces apoptosis $(7,11)$. Results of a large prospective study demonstrated an association between high dietary lignan intake and reduced risk of breast cancer (10). Previously, it was reported that isolation of a unique class of vitexins, neolignan compounds from the seed of Vitex negundo, a Chinese herb (12), and vitexin compound 1 (VB-1), the purified vitexin compound-1 (a neolignan compound), had potent cytotoxic effects in various cancer cell lines (12). However, the molecular mechanisms by which VB-1 induced growth inhibition in HCC cells remain to be clarified.

Constitutive activation of Akt has been shown to be associated with increased cell growth, proliferation and angiogenesis (13-15). Overexpression of Akt has been reported in a variety of human cancers, including HCC. Cells that express elevated level of phosphorylated Akt protein, negatively regulate forkhead transcription family members, in particular forkhead box protein O3a (FOXO3a) (16-19). These FOXO proteins indirectly inhibit cell growth and angiogenesis (20). Findings of recent studies have demonstrated that the ampli- 
fication or activation of Akt is required for the growth and survival of HCC cell lines $(21,22)$, supporting the participation of an activated Akt pathway in the tumorigenesis of HCC.

FOXO3a is a forkhead/winged helix box class O (FOXO) transcription factor that is involved in a variety of cell processes such as cell cycle progression, stress detoxification, DNA damage repair, glucose metabolism and differentiation (23). It is regulated by a number of mechanisms, including phosphorylation. Phosphorylated FOXO3a proteins have been shown to bind to 14-3-3 chaperone proteins causing them to become sequestered in the cytoplasm, where they are unable to regulate gene expression. When active, FOXO3a induces cell cycle arrest and apoptosis, by negatively mediating angiogenesis signaling and acting as an anti-proliferative factor.

Studies in mammalian cells have identified FOXO3a as a target gene that regulates other genes such as survivin, $\mathrm{p} 27^{\mathrm{Kip} 1}$, p21 and vascular endothelial growth factor (VEGF) (24). FOXO3a transcription factors are known to be cellular targets of antitumor drugs used to treat HCC (25) and chronic myeloid leukemia (26). However, whether VB-1 induces cell cycle arrest and angiogenesis inhibition in HCC cells by regulating FOXO3a transcription factor has yet to be determined.

In this study, we investigated the anticancer activity of VB-1 and analyzed its underlying molecular mechanisms. The results demonstrated that VB-1 selectively induces cytotoxicity in HCC cells. It inhibits phosphorylation of Akt and FOXO3a transcription factor, leading to cell cycle arrest and inhibition of angiogenesis.

\section{Materials and methods}

Cell lines and cell culture. Human HCC cell lines Hep3B, Huh-7 and HepG2 and human embryo liver L-02 cells were purchased from the China Centre for Type Culture Collection (CCTCC, Wuhan, China). The cells were maintained in Dulbecco's modified Eagle's medium (DMEM) (Life Technologies, Grand Island, NY, USA) supplemented with $10 \%$ fetal bovine serum (FBS) (Invitrogen-Gibco, Grand Island, NY, USA), $100 \mathrm{U} / \mathrm{ml}$ penicillin and $100 \mathrm{U} / \mathrm{ml}$ streptomycin and were kept in a humidified atmosphere with $5 \% \mathrm{CO}_{2}$ at $37^{\circ} \mathrm{C}$.

Reagents. VB-1 (6-hydroxy-4-(4-hydroxy-3-methoxyphenyl)3-hydro-methyl-7-methoxy-3,4-dihydro-2-naphthaldehyde) was purified from EVn-50, a mixture of lignan compounds from Vitex negundo seed, as described previously (12). 3-(4,5-Dimethylthiazole-2-yl)-2,5-diphenyltetrazolium bromide (MTT), fluorouracil (5-FU), crystal violet, low-gelling point SeaPlaque agarose and propidium iodide (PI) were purchased from Sigma Chemical Co. (St. Louis, MO, USA). Antibodies against phospho-PI3K, PI3K, phospho-Akt, Akt, p21/CIP1, p27/KIP1, cyclin D1, phospho-FOXO3a, FOXO3a and $\beta$-actin were purchased from Cell Signaling Technology, Inc. (Danvers, MA, USA). Enhanced chemiluminescence (ECL) Western Blot Detection reagents were obtained from Amersham Life Sciences, Inc. (Arlington Heights, IL, USA).

MTT assay. Cells were seeded in a 96-well plate at a density of $0.5 \times 10^{4}$ cells/well and maintained in serum-free medium for $24 \mathrm{~h}$. Various concentrations of experimental agents were added to each well and cultured for $48 \mathrm{~h}$, followed by incubation with media containing $0.5 \mathrm{mg} / \mathrm{ml} \mathrm{MTT}$ for $4 \mathrm{~h}$. The supernatant was removed by centrifugation $(1,000 \mathrm{rpm}$, $5 \mathrm{~min})$ DMSO $(100 \mu \mathrm{l})$ was added and absorbance at $570 \mathrm{~nm}$ $\left(\mathrm{A}_{570}\right)$ was measured using an enzyme-labeling instrument (ELX-800 type; Bio-Tek, Shanghai, China). The relative cell viability rate was calculated as: (average $A_{570}$ of the experimental group/average $A_{570}$ of the control group) x $100 \%$.

Clonogenic assay. Cells were plated in 24-well plates at a density of 300 cells/well for $24 \mathrm{~h}$, prior to the addition of various concentrations of experimental agents. After $48 \mathrm{~h}$ the drug-containing medium was removed and replaced with complete growth medium. The medium was then replaced every 3 days for 7-10 days until visible colonies formed. Colonies were simultaneously fixed and stained with $0.5 \%$ crystal violet in methanol. Individual stained colonies in each well were manually counted and the colony formation fraction was calculated as: colony number/(number of cells seeded x plating efficiency). Plating efficiency was estimated as the colony number divided by the number of cells seeded in the drug-free medium.

Soft agar cloning assay. The base layer consisted of $0.6 \%$ lowgelling point SeaPlaque agarose in complete DMEM culture medium. Soft agar consisting of $0.3 \%$ SeaPlaque agarose in complete DMEM culture medium was mixed with $1 \times 10^{4} \mathrm{HCC}$ cells and plated on top of the base layer in a $60 \mathrm{~mm}$ diameter culture dish. Soft agar cultures were maintained at $37^{\circ} \mathrm{C}$ and observed with a Leica DMI 4000B microscope imaging system to evaluate colony counts.

Cell cycle analysis by flow cytometry. Cells were plated in 6 -well plates at a density of $1 \times 10^{6}$ cells/well for $24 \mathrm{~h}$, prior to the addition of various concentrations of test agents. After $24 \mathrm{~h}$ the cells were harvested, and DNA content was stained for $15 \mathrm{~min}$ at $37^{\circ} \mathrm{C}$ with a solution containing $0.4 \%$ Triton X-100 (Sigma), $50 \mu \mathrm{g} / \mathrm{ml}$ of PI (Sigma), and $2 \mu \mathrm{g} / \mathrm{ml}$ of DNase-free RNase (Roche, Indianapolis, IN, USA). The plates were then analyzed for cell cycle perturbation using a FACSCalibur system (FACS 420; Becton-Dickinson, Franklin Lakes, NJ, USA). The CellQuest program was used to quantify the distribution of cells in each cell cycle phase: G1, S and G2/M.

DNA transfection. The small interfering RNA (siRNA) duplexes targeting the sequence 5'-UAAUGUGCCCGUCCUU GUCUU-3' of the human Akt gene, the sequence 5'-ACU CCGGGUCCAGCUCCAC-3' of the FOXO3a gene and control siRNA oligonucleotides were purchased from Dharmacon Research, Inc. (Lafayette, CO, USA). For silencing of Akt or FOXO3a, HepG2 cells were transfected with double stranded siRNA of Akt or FOXO3a using a Signal Silence siRNA kit from Cell Signaling Technology, Inc. Briefly, $1 \times 10^{6}$ cancer cells were plated in $60 \mathrm{~mm}$ Petri dishes for $24 \mathrm{~h}$ and exposed to $3 \mathrm{ml}$ of transfection medium containing $20 \mu \mathrm{g}$ Lipofectamine 2000 and $100 \mathrm{nM}$ siRNA for $24 \mathrm{~h}$. Gene silencing in transfected cells was confirmed by western blotting.

Western blot analysis. Western blot analyses were performed as described previously (12). In brief, cells were lysed in RIPA buffer containing $1 \mathrm{X}$ protease inhibitor cocktail. Protein 
concentrations were determined using the Bradford assay (Bio-Rad, Philadelphia, PA, USA). Proteins were separated using 10-12.5\% SDS/PAGE and transferred to membranes (Millipore, Bedford, MA, USA) in a Tris $(20 \mathrm{mM})$, glycine $(150 \mathrm{mM})$ and methanol $(20 \%)$ buffer at $55 \mathrm{~V}$ for $4 \mathrm{~h}$ at $4^{\circ} \mathrm{C}$. After blocking in 5\% non-fat dry milk in TBS, the membranes were incubated with the primary antibodies at 1:1,000 dilution in TBS overnight at $4^{\circ} \mathrm{C}$. The membranes were then washed three times with TBS-Tween-20, and incubated with secondary antibodies conjugated with horseradish peroxidase at 1:5,000 dilutions in TBS for $1 \mathrm{~h}$ at room temperature. The membranes were washed another three times in TBS-Tween-20 at room temperature. Protein bands were visualized on X-ray film using an enhanced chemiluminescence detection system.

Determination of VEGF. Cells were seeded in 6-well plates $\left(1.0 \times 10^{5}\right.$ cells/well) and incubated at $37^{\circ} \mathrm{C}$. After $24 \mathrm{~h}$, the cell culture supernatant was collected, and the cells were counted after trypsinization. After collection, the medium was centrifuged at $800 \times \mathrm{g}$ for $3 \mathrm{~min}$ at $4^{\circ} \mathrm{C}$ to remove cell debris. The supernatant was frozen at $-20^{\circ} \mathrm{C}$ for subsequent VEGF assay or assayed immediately using commercially available ELISA kits (R\&D Systems, Minneapolis, MN, USA).

Matrigel in vitro endothelial tube formation assay. Cells were cultured in serum-free DMEM for $24 \mathrm{~h}$. The conditioned media were collected, centrifuged, and transferred to fresh tubes and stored at $-20^{\circ} \mathrm{C}$. Growth factor-reduced Matrigel $(125 \mu \mathrm{l})$, was thawed on ice, and plated in a 6-well plate. The plate was then incubated at $37^{\circ} \mathrm{C}$ for $30 \mathrm{~min}$ to allow the Matrigel to polymerize. HUVECs were trypsinized and seeded $\left(5 \times 10^{4}\right.$ cells/well) in each well with $250 \mu 1$ of conditioned medium from VB-1-treated or non-treated control HepG 2 cells. The plate was incubated for $6 \mathrm{~h}$. Each well was photographed using an inverted microscope with digital camera. The assessment of the vessel number of the vessel perimeter in each field was calculated using the Scion Image analysis program.

Statistical analysis. Statistical analysis was performed using Prism statistical analysis software (GrafPad Software, Inc., San Diego, CA, USA). The data are presented as means \pm standard deviations of experiments performed in triplicate for each experimental group. Differences between groups were analyzed by one- or two-way analysis of variance (ANOVA), followed by Bonferoni's multiple comparison tests. $\mathrm{P}<0.05$ was considered to indicate statistical significance.

\section{Results}

VB-1 inhibits the growth of human HCC cells. VB-1 has been shown to inhibit the growth of several cancer cell lines, including HCC cells (12). Therefore, its effects on cell viability in the HCC cell lines HepG2, Hep3B and Huh-7 were examined using an MTT assay. Fig. 1A shows that VB-1 inhibited cell viability in a concentration-dependent manner. The HepG2 cell line was most sensitive, the Hep3B cell line was moderately sensitive, and the Huh-7 cell line was least sensitive. VB-1 had little effect on the human embryo liver L-02 cell line. These data suggested that VB-1 is likely a selective agent that inhibits HCC cell proliferation.
We examined the effects of VB-1 on colony formation of the HCC cell line HepG2 on agar plates and showed that VB-1 inhibited anchorage-dependent growth in a dose-dependent manner (Fig. 1B and C). Additionally VB-1 inhibited colony formation in a dose-dependent manner in a soft agar assay (Fig. 1D and E). These data suggest that VB-1 acts as a potent chemopreventive agent for HCC.

$V B-1$ arrests the cell cycle at the G1/G0 phase in HepG2 cells. It has been previously demonstrated that VB-1 is capable of inducing G1/G0 phase arrest in breast cancer cells (12). Therefore, we investigated whether a similar effect occurs in HepG2 cells. The effect of VB-1 on the cell cycle was determined using FCM analysis. VB-1 resulted in an accumulation of cells in the G1/G0 phase (Fig. 2). This concentration-dependent effect suggests that VB-1 induces cell growth inhibition in HepG2 cells via G1/G0 phase arrest.

VB-1 decreases PI3K and Akt phosphorylation in HepG2 cells. Akt is constitutively active in most cancer cells, and it enhances cell proliferation (27). In order to understand the correlation between PI3K/Akt and VB-1-induced growth inhibition, we determined the expression of protein phosphorylation of PI3K and Akt in cells exposed to VB-1. VB-1 resulted in slight inhibition of PI3K phosphorylation in HepG2 cells (Fig. 3A) and relatively marked inhibition of Akt phosphorylation (Fig. 3B). VB-1 had no effect on total PI3K and Akt expression (Fig. 3A and B). These data suggest that VB-1 inhibits cell proliferation by inhibiting $\mathrm{PI} 3 \mathrm{~K}$ and Akt phosphorylation.

To investigate the role of Akt in VB-1-mediated growth inhibition, we used a siRNA that specifically silences Akt. Expression of siRNAs silence gene expression resulting in functional inactivation (6,7). Western blotting showed that Akt was downregulated after transfection with a siRNA that targeted Akt in HepG2 cells (Fig. 3C). In addition, siRNA Akt enhanced the ability of VB-1 to inhibit cell viability in HepG2 cells (Fig. 3D). These results suggested that VB-1-mediated Akt signaling inhibition may contribute to growth inhibition of HepG2 cells.

$V B-1$ regulates the expression of phosphorylated $\mathrm{FOXO} 3 \mathrm{a}$ and downstream target genes associated with cell cycle regulation. Akt kinase has been shown to regulate the phosphorylation of FOXO3a protein $(28,29)$. The phosphorylation of FOXO3a proteins was determined using western blotting. VB-1 inhibited the phosphorylation of FOXO3a protein. However, VB-1 had no effect on the total protein of FOXO3a expression (Fig. 4A).

Downregulation of FOXO3a by siRNA transfection reduced the expression of FOXO3a protein, as confirmed by western blotting (Fig. 4B). Additionally, VB-1 inhibited the viability of HepG2 cells, and inhibition of FOXO3a expression by siRNA suppressed VB-1-induced growth inhibition (Fig. 4C). These results suggested that VB-1 inhibited growth by regulating $\mathrm{FOXO} 3 \mathrm{a}$.

The effects of VB-1 on cell cycle regulatory genes were also examined. VB-1 induced the expression of the cell cycle inhibitors $\mathrm{p} 21^{\mathrm{CIP} 1}, \mathrm{p} 27^{\mathrm{Kip} 1}$ and inhibited cyclin D1 expression in HepG2 cells (Fig. 4D). These results suggested that VB-1 induced growth arrest by regulating the expression of cell cycle genes. 

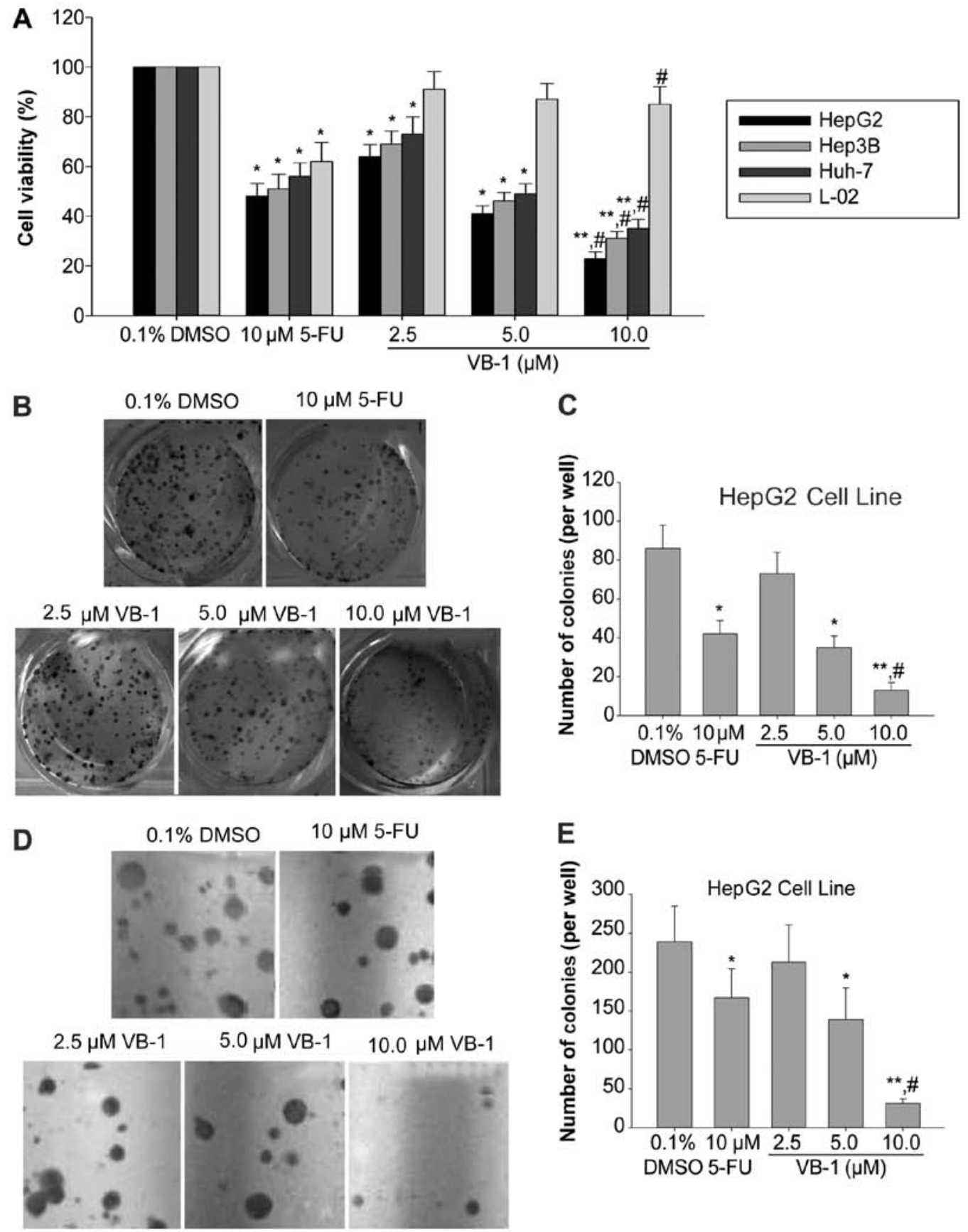

Figure 1. Vitexin compound 1 (VB-1) inhibited the viability of hepatocellular carcinoma (HCC) cells. (A) HepG2, Hep3B, Huh-7 and human embryo liver L-02 cells were exposed to VB-1 (0-10 $\mu \mathrm{M})$ for $48 \mathrm{~h}$. Cell viability was measured by MTT assay. (B and C) VB-1 inhibited the colony formation of the HepG2 cell line. (D and E) VB-1 inhibited the colony formation of the HepG2 cell line in soft agar. Data are shown as means $\pm \mathrm{SD}, \mathrm{n}=3$. $\mathrm{P}<0.05$ compared with respective controls; ${ }^{* *} \mathrm{P}<0.01$ compared with respective controls; ${ }^{*} \mathrm{P}<0.05$ compared with $10 \mu \mathrm{M}$ fluorouracil (5-FU).

Conditioned medium from cells exposed to VB-1 inhibits VEGF activity and tube formation of HUVECs induced. FOXO3a has been shown to regulate VEGF signaling in various cell types (30). To determine whether VB-1 reduces VEGF protein expression and activity by activating FOXO3a, we examined the levels of VEGF protein expression and activity in culture medium. The results showed that VB-1 reduced VEGF protein expression and decreased the levels of VEGF secreted in the culture medium (Fig. 5A and B). However, there was a marked increase in the activity of VEGF in FOXO3a siRNA transfected HepG2 cells (data not shown).
It has been suggested that the activation of FOXO3a transcription factor is an important physiological process that inhibits angiogenesis and ultimately controls tumor growth (31). FOXO3a has been shown to inhibit angiogenesis and metastasis in certain tumor models (22). Since VB-1 inhibited VEGF expression and secretion in HepG2 cells, we examined whether conditioned media from VB-1-treated cells were able to reduce tube formation, which is an indirect measure of angiogenesis. An in vitro tube formation assay was performed in growth factor-reduced Matrigel. Conditioned media from VB-1-treated cells significantly reduced tube 
A
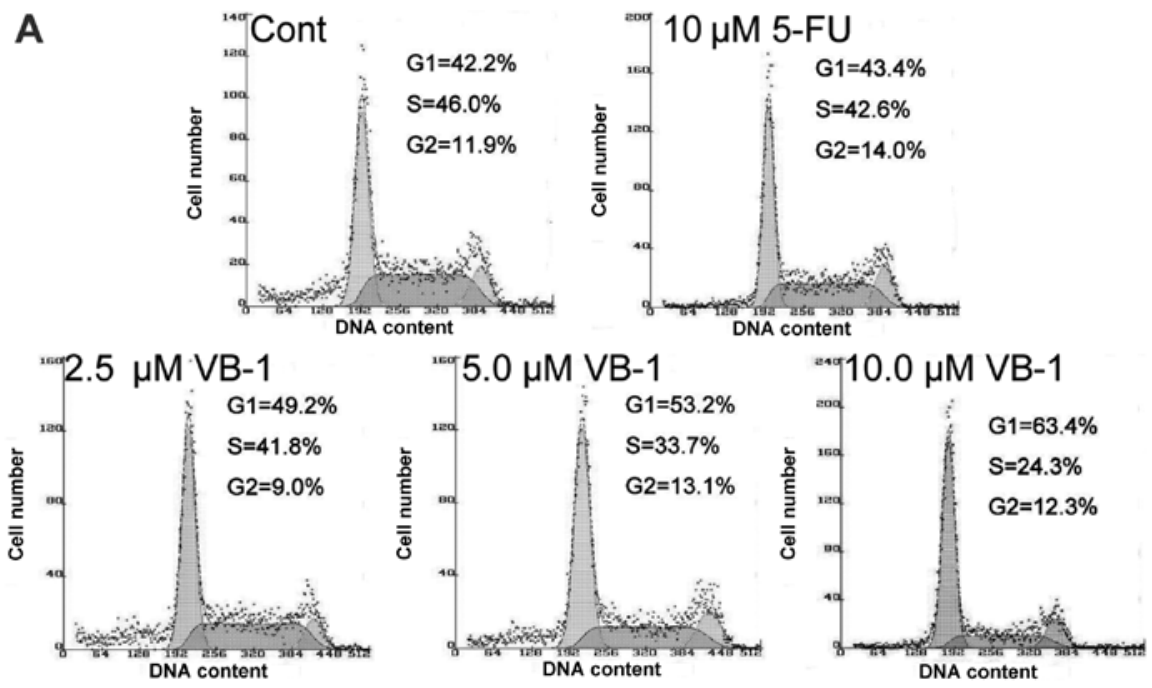

B

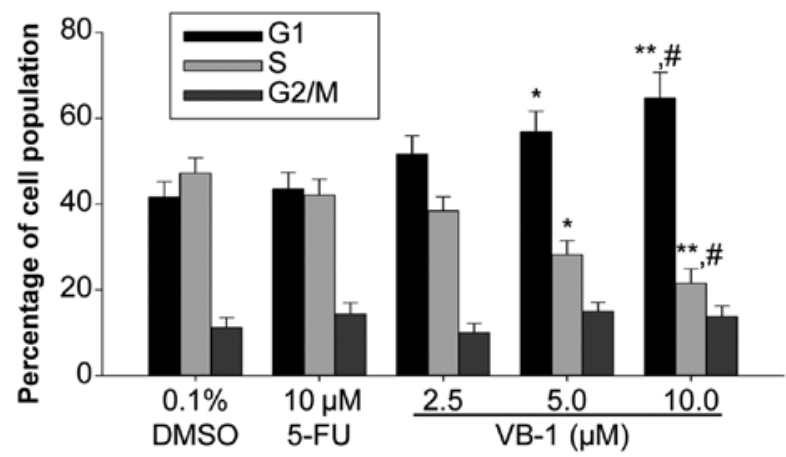

Figure 2. Vitexin compound 1 (VB-1) induced cell cycle arrests in HepG2 cells at G0/G1. (A) HepG2 cells were exposed to VB-1 (0-10 $\mu$ M) for 24 h. The phase distribution of the cell cycle was analyzed using flow cytometry with propidium iodide (PI) staining. (B) Data are shown as means \pm SD, $n=3$. ${ }^{*} \mathrm{P}<0.05$ compared with respective controls; ${ }^{* *} \mathrm{P}<0.01$ compared with respective controls; ${ }^{\#} \mathrm{P}<0.05$, compared with $10 \mu \mathrm{M}$ fluorouracil (5-FU).
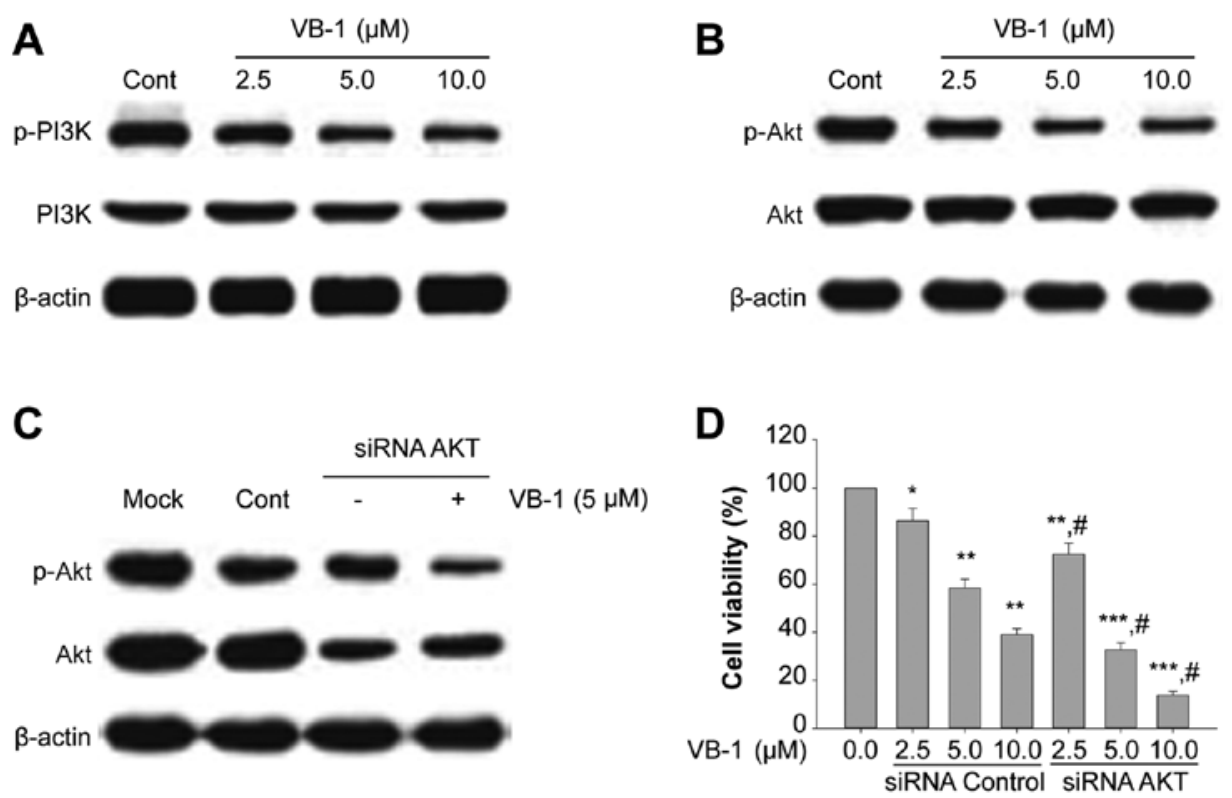

Figure 3. Vitexin compound 1 (VB-1) decreased the phosphorylation levels of PI3K and Akt proteins. (A and B) HepG2 cells were exposed to VB-1 $(0-10 \mu \mathrm{M})$ for $24 \mathrm{~h}$. Western blotting was performed for p-PI3K, total-PI3K, p-Akt, total-Akt and the loading control $\beta$-actin. (C) HepG2 cells were transfected with $100 \mathrm{nM}$ of small interfering RNA (siRNA) control or the siRNA duplexes against Akt mRNA. Twenty-four hours after the transfection, the cells were exposed to $5 \mu \mathrm{M}$ VB-1 for $24 \mathrm{~h}$. Western blotting of p-Akt and Akt was performed to confirm the downregulation of Akt by siRNA transfection. $\beta$-actin was used as the loading control. (D) HepG2 cells were transfected with $100 \mathrm{nM}$ of siRNA control or the siRNA duplexes against Akt mRNA. Twenty-four hours after the transfection, the cells were exposed to VB-1 (0-10 $\mu \mathrm{M})$ for $24 \mathrm{~h}$. Cell viability was examined by MTT assay. ${ }^{*} \mathrm{P}<0.05$ compared with respective controls; ${ }^{* *} \mathrm{P}<0.01$ compared with respective controls; ${ }^{* * *} \mathrm{P}<0.001$ compared with respective controls; ${ }^{*} \mathrm{P}<0.05$ compared with the same concentration of VB-1 in combination with siRNA control. 


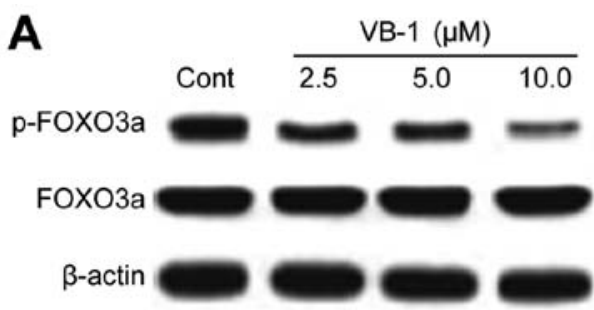

C

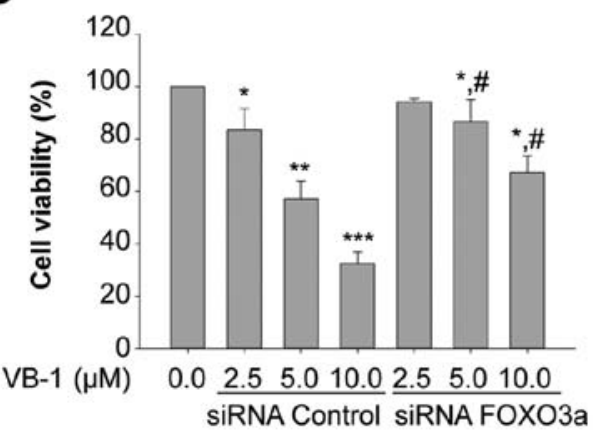

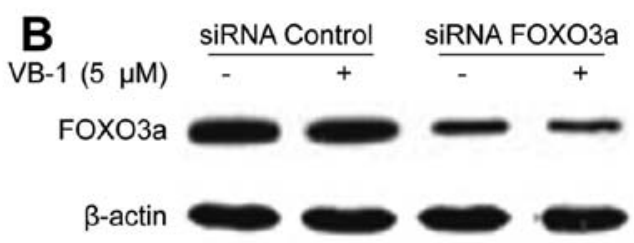

D

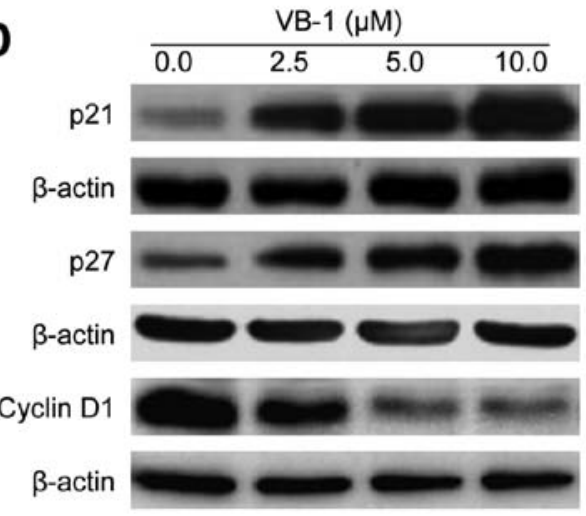

Figure 4. Vitexin compound 1 (VB-1) inhibited forkhead box protein O3a (FOXO3a) phosphorylation and its downstream cell cycle-associated genes. (A) HepG2 cells were exposed to different concentrations of VB-1 for $24 \mathrm{~h}$, and western blotting was undertaken to evaluate the expression of p-FOXO3a, total-FOXO3a and the loading control $\beta$-actin. (B) HepG2 cells were transfected with $100 \mathrm{nM}$ of small interfering RNA (siRNA) control or the siRNA duplexes against FOXO3a mRNA. Twenty-four hours after transfection, the cells were exposed to $5 \mu \mathrm{M}$ VB-1 for $24 \mathrm{~h}$. Western blotting of FOXO3a was undertaken to confirm the downregulation of FOXO3a by siRNA transfection. $\beta$-actin was used as the loading control. (C) HepG2 cells were transfected with $100 \mathrm{nM}$ of siRNA control or the siRNA duplexes against FOXO3a mRNA. Twenty-four hours after the transfection, the cells were exposed to VB-1 (0-10 $\mu \mathrm{M})$ for $24 \mathrm{~h}$. Cell viability was examined by MTT assay. ${ }^{*} \mathrm{P}<0.05$ compared with respective controls; ${ }^{* *} \mathrm{P}<0.01$ compared with respective controls; ${ }^{* * *} \mathrm{P}<0.001$ compared with respective controls; ${ }^{~} \mathrm{P}<0.05$ compared with the same concentration of VB-1 in combination with siRNA control. (D) HepG2 cells were treated with the indicated concentrations of VB-1 for $24 \mathrm{~h}$. Western blotting was undertaken to investigate the expression of p21, p27, cyclin D1 and the loading control $\beta$-actin.

A
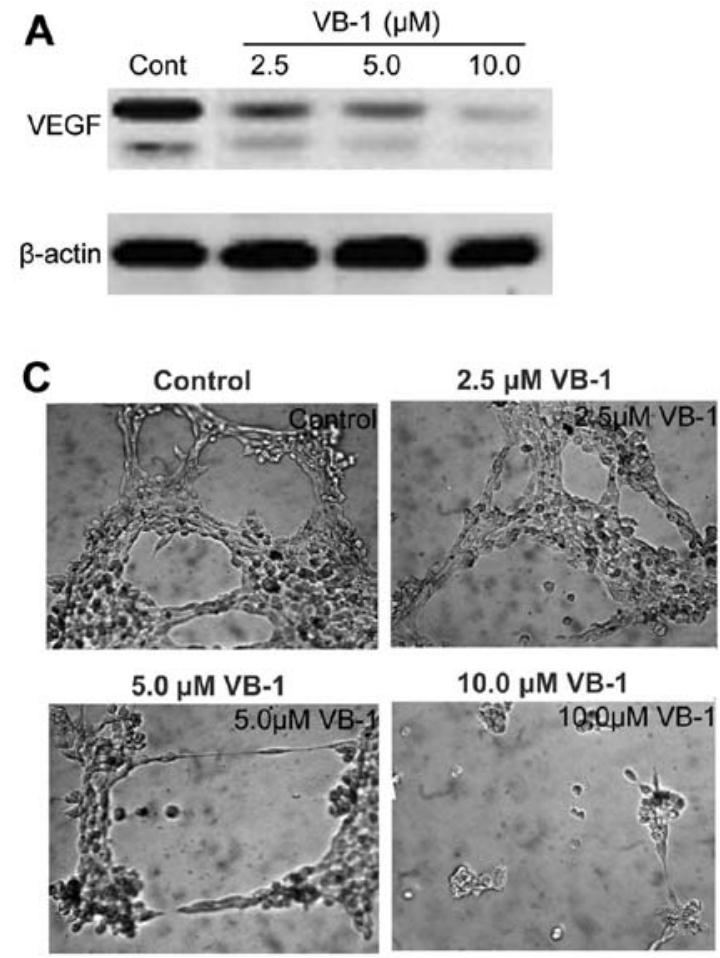

$10.0 \mu \mathrm{M}$ VB-1

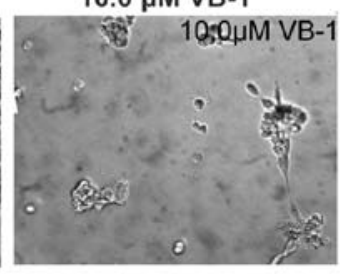

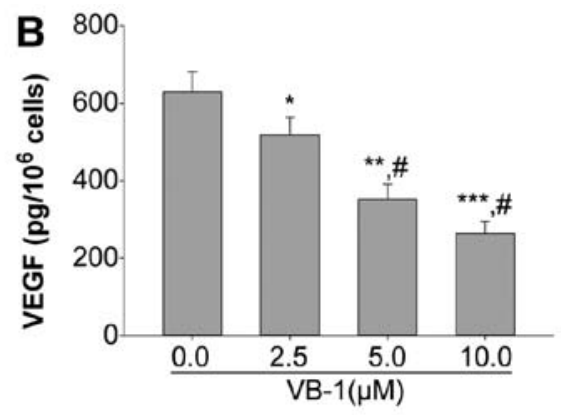

D

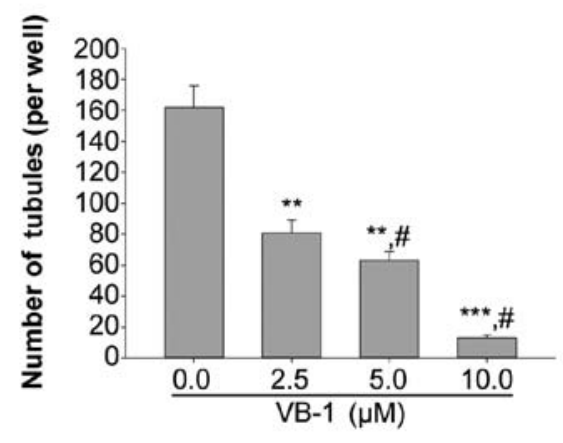

Figure 5. Vitexin compound 1 (VB-1) inhibited vascular endothelial growth factor (VEGF) expression and the angiogenic potential of HepG2 cells. (A) HepG2 cells were treated with the indicated concentrations of VB-1 for $24 \mathrm{~h}$, and western blotting of VEGF and the loading control $\beta$-actin was performed. (B) VB-1 inhibited the activity of VEGF in HepG2 cells. The experiments were repeated three times. ${ }^{*} \mathrm{P}<0.05,{ }^{* *} \mathrm{P}<0.01$ and ${ }^{* * *} \mathrm{P}<0.001$, relative to control; ${ }^{*} \mathrm{P}<0.05$ compared with $2.5 \mu \mathrm{M}$ VB-1. (C) Conditioned medium from VB-1-treated HepG2 cells significantly reduced tube formation of HUVECs after $6 \mathrm{~h}$ incubation compared with the control medium. (D) Quantification of tube formation of endothelial cells. Image analysis of tubules and capillaries was carried out using software image analysis program Scion Image. ${ }^{* *} \mathrm{P}<0.01,{ }^{* * *} \mathrm{P}<0.001$ relative to control, ${ }^{,} \mathrm{P}<0.05$ compared with $2.5 \mu \mathrm{M}$ VB-1. 
formation of HUVECs after $6 \mathrm{~h}$ incubation compared with the medium from control cells (Fig. 5C and D). By contrast, conditioned media from FOXO3a siRNA-transfected HepG2 cells increased tube formation of HUVECs after $6 \mathrm{~h}$ incubation compared with the medium from control siRNA-transfected cells (data not shown).

\section{Discussion}

Surgery, chemotherapy, and radiotherapy are generally used to treat HCC, however, no effective therapy for advanced HCC is currently available. Thus, there is a need to identify other therapeutic agents against this disease. VB-1, the purified vitexin compound 1 present in Vitex negundo seed, has been shown to exert anticancer activity and to be associated with low toxicity (12). In the present study, we showed that VB-1 suppressed the proliferation of HCC cell lines HepG2, Hep3B, Huh-7, but had little effect on L-02 cells. Additionally, VB-1 significantly inhibited the anchorage-dependent and anchorage-independent growth of HepG2 cells in a concentration-dependent manner by inducing cell cycle arrest at G1/G0. VB-1 also reduced the secretion of VEGF, resulting in inhibition of the endothelial tube formation. To the best of our knowledge, we have shown for the first time that VB-1-induced growth inhibition and cell cycle arrest are mediated by regulation of the Akt/FOXO3a pathway.

The Akt pathway is crucial in sustaining survival against the programmed death in cancer cells. It has been widely shown that hyperactivation of Akt is a common event in many human cancer types, and activation of the PI3K/Akt pathway has been reported to contribute to chemotherapy resistance (32-34). This pathway, therefore, is an important target for anticancer therapies. Identification of a safe and effective therapeutic inhibitor of PI3K or Akt continues to be a challenge. This study has demonstrated that VB-1 inactivates Akt, which, in turn, reduces the phosphorylation of FOXO3a, leading to an increased expression of downstream target genes, such as p21 and p27, and a decreased expression of cyclin D1 exprotein. Upregulation of p21 and p27, which bind to CDK2/CD4, have previously been shown to sequester the procedure involved in cell cycle arrest $(35,36)$. This was also demonstrated in our study using VB-1-treated HepG2 cells. In-depth studies are required to clarify whether VB-1 is a direct or indirect inhibitor of Akt. However, the pharmacological and toxicological profiles of VB-1 partly revealed in this study, as well as its in vivo effectiveness in inhibiting xenografted tumor growth (12), suggest that the compound may become an antiAkt candidate for HCC therapy.

In conclusion, taken together our results demonstrate that VB-1 induces cell cycle arrest and growth inhibition by the inactivation of Akt which in turn regulates a FOXO3a transcription factor. Genetic inhibition of Akt pathways are known to have synergistic effects on the activation of FOXO3a transcription factor through dephosphorylation. Thus, VB-1 appears to be an attractive agent for the prevention and treatment of HCC.

\section{Acknowledgements}

We express our thanks to Dr Jian-Guo Cao (Medical College, Hunan Normal University, Changsha, Hunan, China) for critical input into the scientific content of the manuscript. This study was supported by the Major State Science and Technology Special Purpose of China (2009ZX09102-109), the National Science and Technology Major Projects for 'Major New Drugs Innovation and Development' (2012ZX09303014001), the International Science and Technology Cooperation Program of China (2011DFA30620) and the Hunan Province Science and Technology Project (2009FG3142).

\section{References}

1. Jemal A, Bray F, Center MM, Ferlay J, Ward E and Forman D: Global cancer statistics. CA Cancer J Clin 61: 69-90, 2011.

2. Okuda K: Hepatocellular carcinoma. J Hepatol 32: 225-237, 2000.

3. Llovet JM, Burroughs A and Bruix J: Hepatocellular carcinoma. Lancet 362: 1907-1917, 2003.

4. Ferlay J, Shin HR, Bray F, Forman D, Mathers C and Parkin DM: Estimates of worldwide burden of cancer in 2008: GLOBOCAN 2008. Int J Cancer 127: 2893-2917, 2010.

5. Lai EC and Lau WY: The continuing challenge of hepatic cancer in Asia. Surgeon 3: 210-215, 2005.

6. Thompson LU, Chen JM, Li T, Strasser-Weippl K and Goss PE: Dietary flaxseed alters tumor biological markers in postmenopausal breast cancer. Clin Cancer Res 11: 3828-3835, 2005.

7. Park JB, Lee MS, Cha EY, et al: Magnolol-induced apoptosis in HCT-116 colon cancer cells is associated with the AMP-activated protein kinase signaling pathway. Biol Pharm Bull 35: 1614-1620, 2012.

8. Kuijsten A, Arts IC, Hollman PC, van't Veer P and Kampman E: Plasma enterolignans are associated with lower colorectal adenoma risk. Cancer Epidemiol Biomarkers Prev 15: 1132-1136, 2006.

9. Adlercreutz H: Lignans and human health. Crit Rev Clin Lab Sci 44: 483-525, 2007.

10. Bergman Jungestrom $M$, Thompson LU and Dabrosin C: Flaxseed and its lignans inhibit estradiol-induced growth, angiogenesis, and secretion of vascular endothelial growth factor in human breast cancer xenografts in vivo. Clin Cancer Res 13: 1061-1067, 2007.

11. Cuca LE, Coy ED, Alarcón MA, Fernández A and Aristizábal FA: Cytotoxic effect of some natural compounds isolated from Lauraceae plants and synthetic derivatives. Biomedica 31: 335-343, 2011.

12. Zhou Y, Liu YE, Cao J, et al: Vitexins, nature-derived lignan compounds, induce apoptosis and suppress tumor growth. Clin Cancer Res 15: 5161-5169, 2009.

13. Liu Z, Xiao Y, Yuan Y, et al: Effects of oleic acid on cell proliferation through an integrin-linked kinase signaling pathway in 786-O renal cell carcinoma cells. Oncol Lett 5: 1395-1399, 2013.

14. Vene R, Benelli R, Minghelli S, Astigiano S, Tosetti F and Ferrari N: Xanthohumol impairs human prostate cancer cell growth and invasion and diminishes the incidence and progression of advanced tumors in TRAMP mice. Mol Med 18: 1292-1302, 2012

15. Rubinfeld H, Cohen-Kaplan V, Nass D, et al: Heparanase is highly expressed and regulates proliferation in GH-secreting pituitary tumor cells. Endocrinology 152: 4562-4570, 2011.

16. Wu J, Wu X, Zhong D, Zhai W, Ding Z and Zhou Y: Short hairpin RNA (shRNA) Ether à go-go 1 (Eag1) inhibition of human osteosarcoma angiogenesis via VEGF/PI3K/AKT signaling. Int J Mol Sci 13: 12573-12583, 2012.

17. Khaidakov M and Mehta JL: Oxidized LDL triggers pro-oncogenic signaling in human breast mammary epithelial cells partly via stimulation of MiR-21. PLoS One 7: e46973, 2012.

18. van Dijk M, van Bezu J, van Abel D, et al: The STOX1 genotype associated with pre-eclampsia leads to a reduction of trophoblast invasion by alpha-T-catenin upregulation. Hum Mol Genet 19: 2658-2667, 2010.

19. Biggs WH III, Meisenhelder J, Hunter T, Cavenee WK and Arden KC: Protein kinase B/Akt-mediated phosphorylation promotes nuclear exclusion of the winged helix transcription factor FKHR1. Proc Natl Acad Sci USA 96: 7421-7426, 1999.

20. Band AM, Björklund $\mathrm{M}$ and Laiho $\mathrm{M}$ : The phosphatidylinositol 3-kinase/Akt pathway regulates transforming growth factor-\{beta\} signaling by destabilizing ski and inducing Smad7. J Biol Chem 284: 35441-35449, 2009. 
21. Wang L, Jia D, Duan F, et al: Combined anti-tumor effects of IFN-alpha and sorafenib on hepatocellular carcinoma in vitro and in vivo. Biochem Biophys Res Commun 422: 687-692, 2012.

22. Vanbrocklin MW, Robinson JP, Lastwika KJ, McKinney AJ, Gach HM and Holmen SL: Ink4a/Arf loss promotes tumor recurrence following Ras inhibition. Neuro Oncol 14: 34-42, 2012.

23. Kim BC: FoxO3a mediates transforming growth factor-beta1induced apoptosis in $\mathrm{FaO}$ rat hepatoma cells. BMB Rep 41: 728-732, 2008

24. Wang S, Chong ZZ, Shang YC and Maiese K: WISP1 neuroprotection requires FoxO3a post-translational modulation with autoregulatory control of SIRT1. Curr Neurovasc Res 10: 54-69, 2013.

25. Liang C, Chen $\mathrm{W}$, Zhi X, et al: Serotonin promotes the proliferation of serum-deprived hepatocellular carcinoma cells via upregulation of FOXO3a. Mol Cancer 12: 14, 2013.

26. Naka K, Hoshii T, Muraguchi T, et al: TGF-beta-FOXO signalling maintains leukaemia-initiating cells in chronic myeloid leukaemia. Nature 463: 676-680, 2010.

27. Yang Y, Jiang $\mathrm{H}, \mathrm{Gao} \mathrm{H}$, et al: The monoclonal antibody $\mathrm{CH} 12$ enhances the sorafenib-mediated growth inhibition of hepatocellular carcinoma xenografts expressing epidermal growth factor receptor variant III. Neoplasia 14: 509-518, 2012.

28. Chung YW, Kim HK, Kim IY, Yim MB and Chock PB: Dual function of protein kinase $\mathrm{C}$ (PKC) in 12-O-tetradecanoylphorbol13-acetate (TPA)-induced manganese superoxide dismutase (MnSOD) expression: activation of CREB and FOXO3a by PKC-alpha phosphorylation and by PKC-mediated inactivation of Akt, respectively. J Biol Chem 286: 29681-29690, 2011.
29. Kashiwagi A, Fein MJ and Shimada M: Calpain modulates cyclin-dependent kinase inhibitor 1B (p27(Kip1)) in cells of the osteoblast lineage. Calcif Tissue Int 89: 36-42, 2011.

30. Dormond O, Madsen JC and Briscoe DM: The effects of mTOR-Akt interactions on anti-apoptotic signaling in vascular endothelial cells. J Biol Chem 282: 23679-23686, 2007.

31. Davis R, Singh KP, Kurzrock R and Shankar S: Sulforaphane inhibits angiogenesis through activation of FOXO transcription factors. Oncol Rep 22: 1473-1478, 2009.

32. Wu HH, Wu JY, Cheng YW, et al: cIAP2 upregulated by E6 oncoprotein via epidermal growth factor receptor/phosphatidylinositol 3-kinase/AKT pathway confers resistance to cisplatin in human papillomavirus 16/18-infected lung cancer. Clin Cancer Res 16: 5200-5210, 2010.

33. Kreutzer JN, Ruzzene M and Guerra B: Enhancing chemosensitivity to gemcitabine via RNA interference targeting the catalytic subunits of protein kinase CK2 in human pancreatic cancer cells. BMC Cancer 10: 440, 2010.

34. Fleischer B, Schulze-Bergkamen H, Schuchmann M, et al: Mcl-1 is an anti-apoptotic factor for human hepatocellular carcinoma. Int J Oncol 28: 25-32, 2006.

35. He L, Yang X, Cao X, Liu F, Quan M and Cao J: Casticin induces growth suppression and cell cycle arrest through activation of FOXO3a in hepatocellular carcinoma. Oncol Rep 29: 103-108, 2013.

36. Nayak G and Cooper GM: p53 is a major component of the transcriptional and apoptotic program regulated by PI 3-kinase/Akt/ GSK3 signaling. Cell Death Dis 3: e400, 2012. 